\title{
A NOVEL HYBRID COMMUNICATION TECHNIQUE INVOLVING POWER LINE COMMUNICATION AND VISIBLE LIGHT COMMUNICATION
}

\author{
Bindu Elias ${ }^{1}$, Arjun $S^{2}$, Athul R $\mathbf{S}^{3}$, Basil Paul ${ }^{4}$, Nidhun Das $\mathbf{P}^{5}$ \\ ${ }^{1}$ Professor [EEE], Dept. of Electrical and Electronics, Mar Athanasius College of Engineering, Kothamangalam, India \\ ${ }^{2} U G$ Student [EEE], Dept. of Electrical and Electronics, Mar Athanasius College of Engineering, Kothamangalam, India \\ ${ }^{3} U G$ Student [EEE], Dept. of Electrical and Electronics, Mar Athanasius College of Engineering, Kothamangalam, India \\ ${ }^{4} U G$ Student [EEE], Dept. of Electrical and Electronics, Mar Athanasius College of Engineering, Kothamangalam, India \\ ${ }^{5}$ UG Student [EEE], Dept. of Electrical and Electronics, Mar Athanasius College of Engineering, Kothamangalam, India
}

\begin{abstract}
Power Line Communication (PLC) is a technology that employs the infrastructure of electrical power distribution system as communication medium. PLC technology could provide the consumer with a spectrum of services such as Internet, home entertainment and home automation. Dealing with very high voltages and its isolation is a problem of PLC. A solution to this problem is proposed by combining Power Line Communication and Visible Light Communication. Visible Light Communication is a mode of communication which uses visible light as transmission medium. ASK modulated signal is used to control the switching of LED bulbs which are connected to the same power line. This serves the dual purpose of lighting and transmitter of Visible Light Communication. This light is allowed to fall on photodiodes and the output of photodiodes is used to recreate the original signal. This system has numerous advantages. Since it doesn't use radio frequencies it can be implemented in RF sensitive environments such as hospitals, airplanes etc. The extra cost required in the infrastructure development is comparatively less.
\end{abstract}

Keywords: Visible Light Communication, Power Line Communication ****

\section{INTRODUCTION}

Power line communication is the art of sending data through power lines. It can provide spectrum of services such as internet, home automation and home entertainment. Major problem associated with this is the isolation of power line. One of the solutions for the above problem is integrating power line with visible light communication. LED is more advantageous than the existing incandescent lamp in terms of long life expectancy, high tolerance to humidity, low power consumption and minimal heat generation [1]. LED is used in full colour displays, traffic signals and many other means of illumination. In this system LED is not only used as a lighting device but also used as a communication device. It is a kind of optical wireless communication that uses the "visible" white ray as the medium. This dual function of LED for lighting and communication emerges many new and interesting applications. The function is based on the fast switching of LEDs and the modulation of the visible-light waves for freespace communications [1]. The advantages of the proposed system when compared with infrared wireless communication are LED light has higher power thus receiver terminal does not need to narrow the angle, the shadowing can be minimized because LED lights are distributed within a room, the installation is easy and LED is aesthetically pleasing, they do not cause or suffer from radio or electromagnetic interference. So it can be used in RF sensitive environment such as hospitals, airplane etc. In the proposed system the signal to be transmitted is sent through the power line. It is modulated before reaching on visible light transmitter. The modulated signal is picked up by the transmitter. LED will convert the electrical signal into optical signal. Photodiode receives these signals and convert back into the electrical signal. It is demodulated according to the intensity of received light. 


\section{PROPOSED SYSTEM DESCRIPTION}

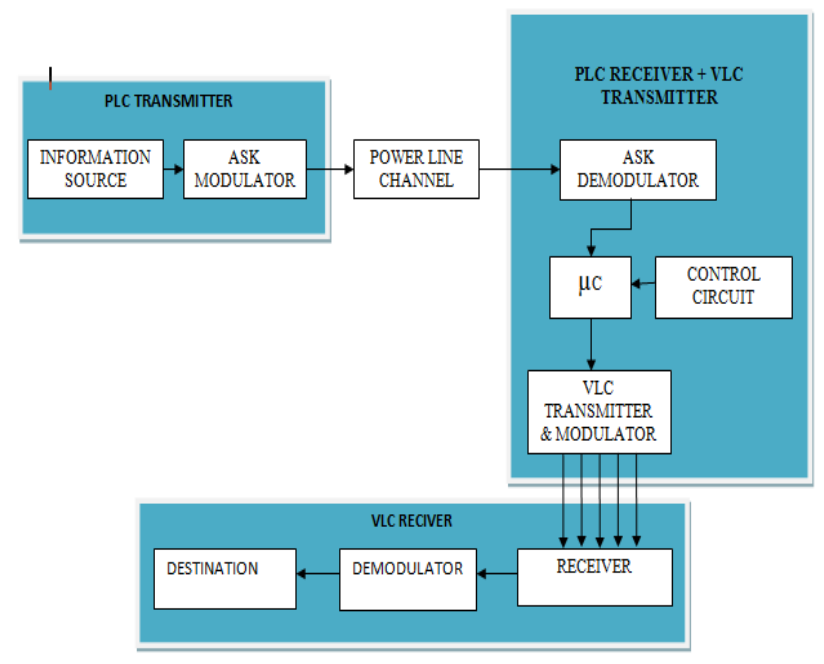

Fig 1: General block diagram of proposed system

The system mainly consists of power line and visible light section. PLC employs the infrastructure of electrical power distribution system as communication medium. Power line modem is plugged into the existing power line network and the ASK modulated signal is transmitted through it. This signal is captured by the PLC receiver and it is demodulated. This is routed to the visible light section using microcontroller. FSK modulation is used in VLC and the communication is simplex. Here LED will convert the electrical signal into optical signal which is received using a photodiode and convert back into electrical signal.

\subsection{Power Line Communication}

In power line communication data is sent through power line. Basic processes involved are modulation, transmit the signal in such a manner it reduce signal distortion, demodulation and routing it to the visible light section. Power line modem can perform the above functions.

Basically a power line modem consists of a pic microcontroller, a carrier frequency oscillator, a digital modulator and an interfacing circuit. The pic microcontroller accepts the serial data transmitted from server, through a level shifter the data is given to an OOK (ON OFF Key) modulator which performs ASK modulation. In this modulation technique the modulator transmits carrier signal for transmission of digital data 'one' and no carrier signal is present during the transmission of a 'zero'. A carrier frequency oscillator of frequency $400 \mathrm{kHz}$ [4] is used to produce a high frequency carrier signal for minimizing distortion. The modulated signal is loaded to power line through an interfacing circuit consisting of data/voice coupling transformer MAX240. The interfacing circuit is used to isolate the $230 \mathrm{~V} / 50 \mathrm{~Hz}$ from the low voltage environment. The data transmitted through power line is received at another server through a power line modem which acts as a demodulator. The received signal is amplified and given to an OOK demodulator which reconstructs original digital data. The receiver unit is isolated from power line using an interfacing circuit consists of a data/voice coupling transformer.

\subsection{Visible Light Communication}

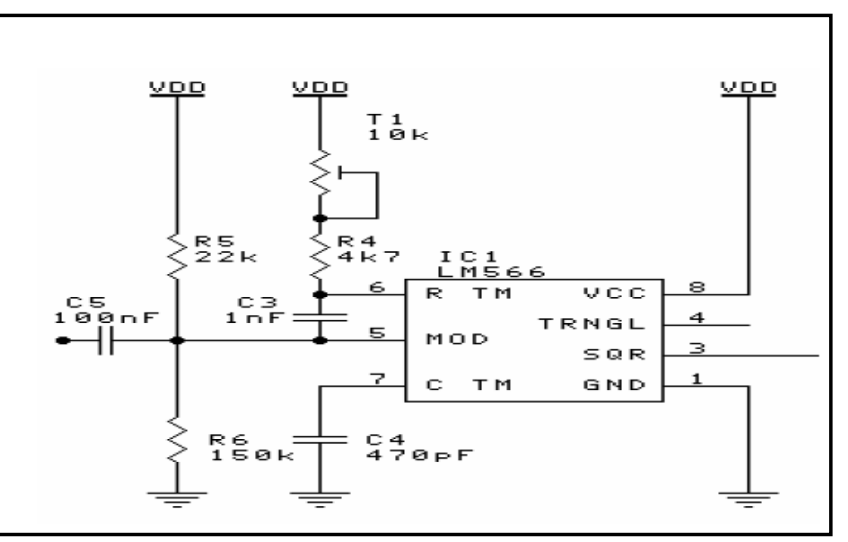

Fig 2: Modulation circuit of Transmitter

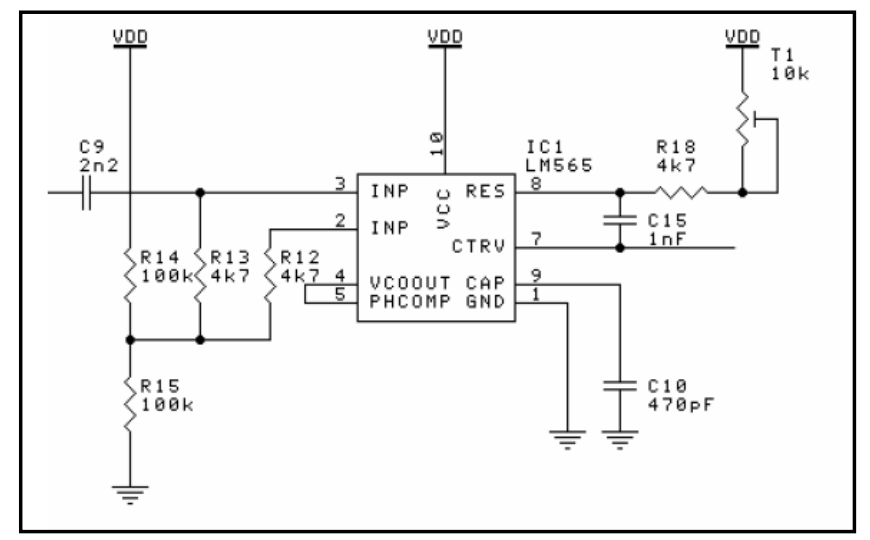

Fig 3: Demodulation circuit of Receiver

The data sent through the power line is routed to the visible light section by the microcontroller. It consists of a modulation stage, transmitter section, receiver section, demodulation stage. Voltage Controlled Oscillator is used to modulate the input signal. Input signal to be superimposed is given to the control voltage pin of voltage controlled oscillator. The VCO is a general purpose voltage controlled oscillator which may be used to generate square and triangular waves, the frequency of which is a very linear function of a control voltage. The frequency is also a function of an external resistor and capacitor. Control pin of VCO is connected to a timing resistor and potentiometer, which works with the timing capacitor connected with VCO to control the modulation frequency. The potentiometer allows to fine tune 
the carrier frequency to produce the best modulated signal. Output of VCO which gives frequency modulated wave. Chosen the values of the timing capacitor and timing resistor so that a carrier signal would have a frequency around $70 \mathrm{kHz}$ [6]. This high frequency FSK modulated signal after amplification is given to the driver of VLC transmitter. FSK modulation is employed in VLC transmission, there by eliminating the flickering of LED and then maintaining the quality of lighting system. LED will convert electrical signal into optical signal. This is received by the photodiode, which will convert back into the original message signal.

The output of photodiode is now passed through the demodulation stage to retrieve the data. It includes the signal amplification circuit, signal demodulation circuit and filters. Frequency modulation is used to modulate the input signal. This frequency modulated signal is recovered using a phase locked loop chip. The general purpose phase locked loop containing a stable, highly linear voltage controlled oscillator for low distortion FM demodulation.
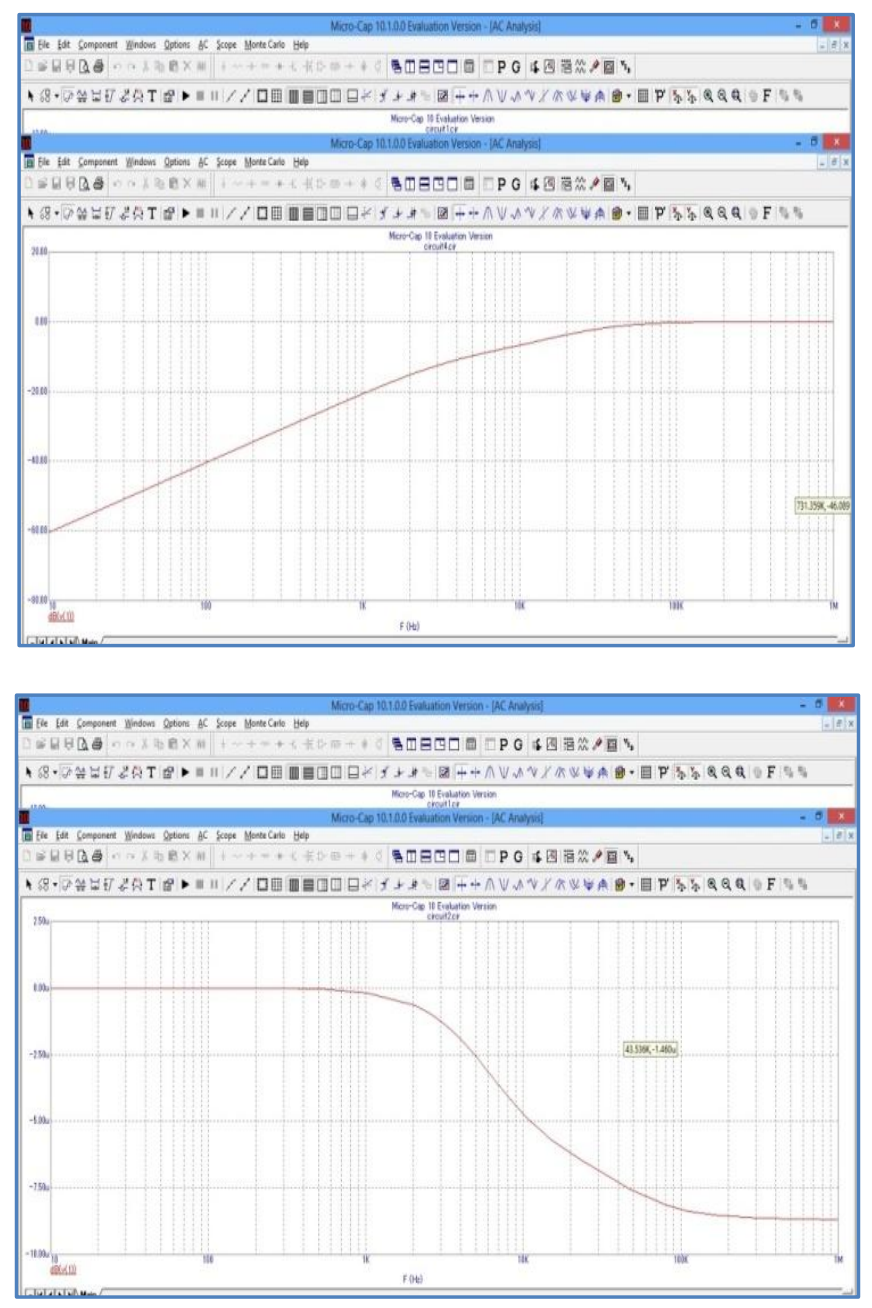

Fig 4: Simulation of Band pass filter

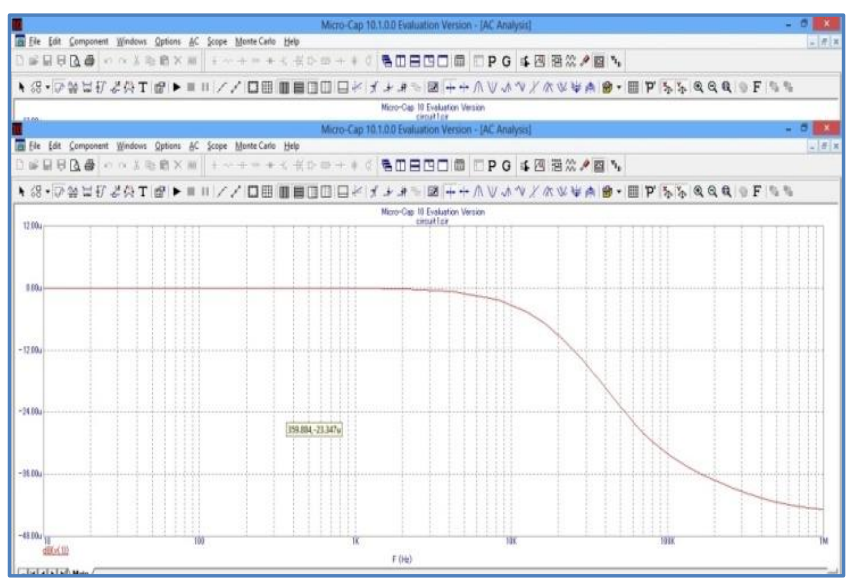

Fig 5: Simulation of low pass filter

\subsection{Architecture}

The topology adopted for the communication system is a hybrid one using bus and tree topology. The power line act as the main bus. Communication through the main bus can be either simplex or half duplex. A number of nodes can be connected to the network. Here only two nodes is connected to the main bus. One of these nodes is the master from which data is sent and the other is an Arduino microcontroller which acts as a router. The data through power line is demodulated and received in this microcontroller. This data is then passed on to the visible light communication section. This section is designed in the form of an inverted tree and simplex communication is employed.

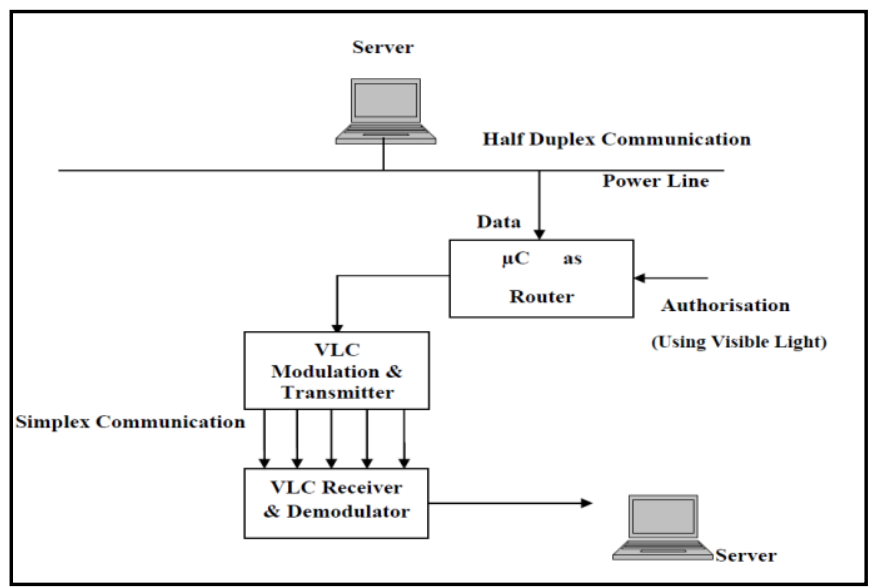

Fig 6: Architecture

\subsection{Authentication}

The retransmission through the Arduino is an authenticated process. For the authentication an Android application is developed which uses the camera flash of the mobile phone to transmit the authentication code. Figure shows the flow chart of sequence of operation. The program code asks the user to 
enter the user name and password. If the entered values are correct the camera flash is activated and made to blink in a particular pattern. The user has the option to authenticate as well as deny the router to retransmit the data. If the user name or password is wrong no action will be performed. In addition to this a message is displayed on screen to inform the user about the accuracy of entered values.

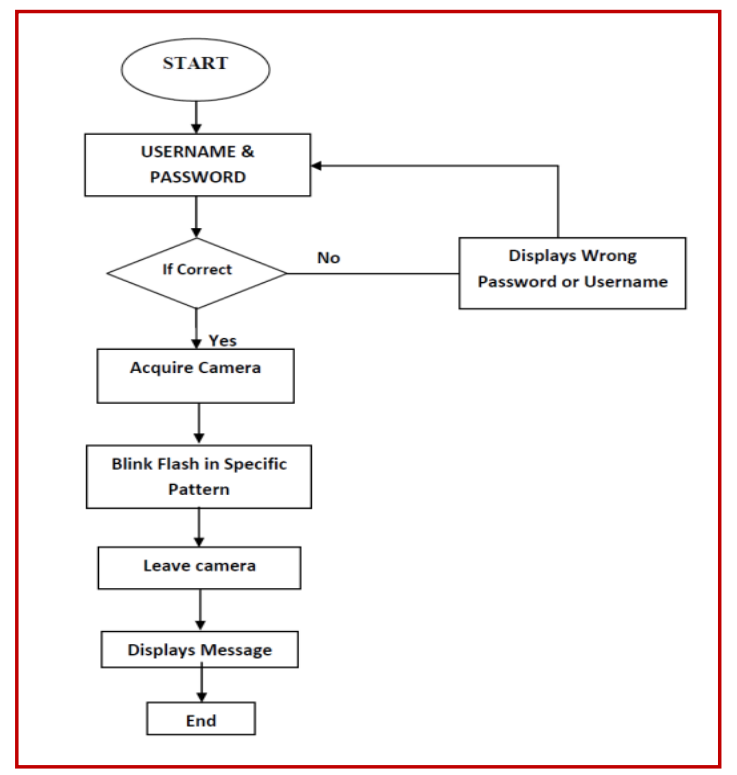

Fig 7: Flow chart for android application

Figure shows the flowchart of router program. The Arduino receives the pattern from camera flash through a receiver similar to visible light receiver. This pattern is compared with one stored in microcontroller's internal memory. If the pattern is correct the microcontroller performs the appropriate operation.

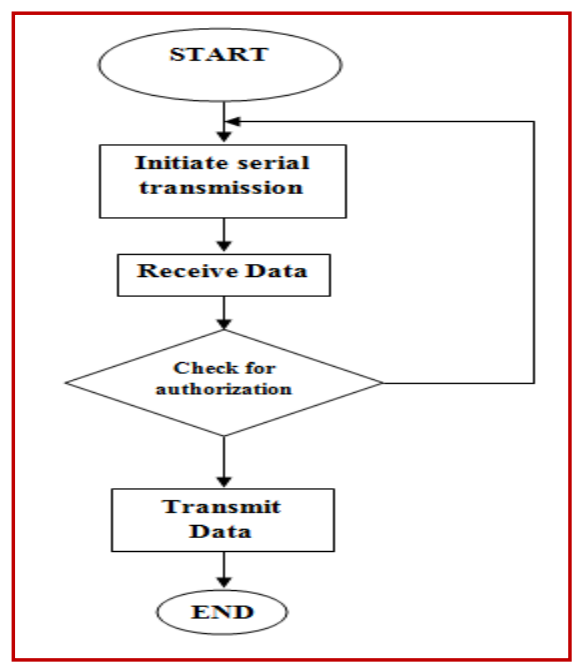

Fig 8: Flow chart for routing program

\section{CONCLUSIONS}

In optical wireless communication systems employing white LED lighting, it is very difficult for existing offices and households to install the communication cable to the ceiling. In this paper, easy wiring system for optical communication using existing power-line is proposed. The composition of this system is very simple. It is very easy to install and also they do not cause radio or electromagnetic interference. This integrated system will surely have a great impact as a new signal transmission system and its economical impact will be great.

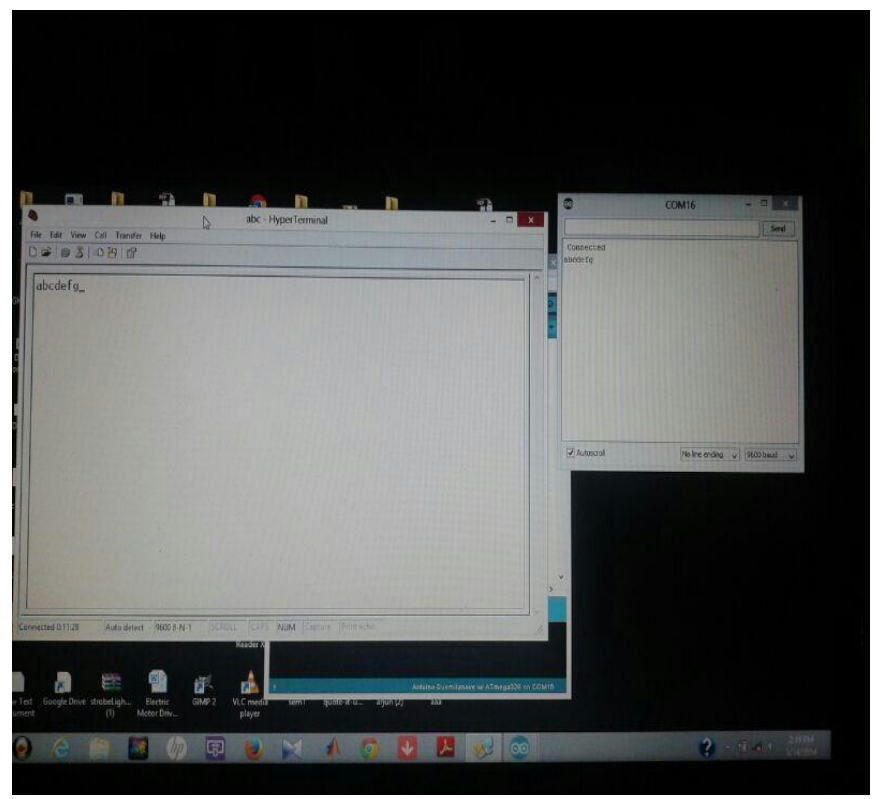

Fig 9: Result of data transmission and reception

\section{REFERENCES}

[1]. Toshihiko Komine, Student Member, IEEE and Masao Nakagawa, Member, IEEE “ Integrated System of White LED Visible-Light Communication and Power-Line Communication

[2]. C. P. Kno, R. M. Fletcher, T. D. Owentowski, M.C. Lardizabal and M.G. Craford, "High performance ALGaInP visible light-emitting diodes,"Appl. Phys. Lett., vol. 57, no.27, pp.2937-2939, 1990.

[3]. T. Komine and M. Nakagawa , “Fundamental Analysis for Visible-Light Communication System Using LED Lights ", IEEE Transactions on Consumer Electronics, Vol. 50, no. 1, pp. 100-107, February 2004

[4]. Masaaki Katayama, "Introduction to Robust, Reliable, and High-speed Power-Line Communication Systems," IEICE Trans. Fundamentals, vol.E84-A, no.12, December 2001

[5]. F.J. Canete, L. Diez, J.A. Cortes, J.T. Entrambasaguas, "Broadband modelling of indoor power-line channels Consumer Electronics," ZEEE Trans. on, vol. 48, no. 1, pp. 175-183, 2002. 
[6]. T. Hara, S. Iwasaki, T. Yendo, T. Fujji, and M. Tanimoto, "A New Receiving System of Visible Light Communication for ITS”, in Proc. IEEE IVS'07, June 2007, Istanbul Turkey. pp. 474-479.

[7]. N. Kumar, L. A. Nero and R. L. Aguiar, "Visible Light Communication for Advanced Driver Assistant Systems", The work is part of FCT project VIDAS - PDTC/EEATEL/75217, 2006. 